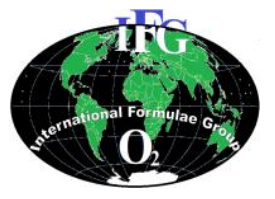

\title{
Etude de quelques paramètres écologiques des Elapidae de six villes de Côte d'Ivoire
}

\author{
Marc Hermann AKAFFOU ${ }^{1,2^{*}}$, Mathieu Tih KOUE BI ${ }^{1}$ et \\ Hilaire Kouassi YAOKOKORE- BEÏBRO ${ }^{1}$ \\ ${ }^{1}$ Laboratoire de Zoologie et Biologie Animale, Université Félix Houphouët Boigny de Cocody, Abidjan, Côte \\ d'Ivoire, 22 B.P.582 Abidjan 22, Côte d'Ivoire. \\ ${ }^{2}$ Unité d'Entomologie et d'Herpétologie, Institut Pasteur de Côte d'Ivoire, 01 B. P. 490 Abidjan 01. \\ *Auteur correspondant ; E-mail : akafpasteur@gmail.com; Tél : (+225) 58917650.
}

\section{RESUME}

Les Elapidae sont souvent incriminés dans des accidents à issue parfois fatale lors de rencontres inopportunes avec l'homme en Côte d'Ivoire. Pourtant, peu d'études sur leur écologie ont été réalisées dans ce pays pour définir des mesures prophylactiques contre ces accidents. Pour ce faire, des milieux anthropisés de six villes de Côte d'Ivoire ont fait l'objet d'un suivi herpétologique régulier de janvier 2014 à décembre 2015. Durant cette période, les captures à la main et le piégeage ont permis l'inventaire de cinq espèces d'Elapidae dans les six villes. Au total, 725 individus ont été capturés dont 299 répartis en cinq espèces dans les jachères, 272 de quatre espèces dans les habitations et 154 individus de trois espèces dans les plantations. Cette faune ophidienne est caractérisée par deux espèces constantes et trois espèces accidentelles. Au regard de l'habitat préférentiel, ces cinq espèces se répartissent en trois espèces mixtes, une espèce de savane et une espèce de forêt. Quatre espèces sont classées dans la catégorie non évaluée de la liste rouge de l'UICN. En considérant le moment nycthéméral de capture, Dendroaspis viridis a été abondamment obtenu le jour. Ces quelques paramètres écologiques sur les Elapidae à présent mieux connus se révèlent d'un grand intérêt pour l'évaluation du risque épidémiologique des morsures par Elapidae dans les villes étudiées.

() 2019 International Formulae Group. All rights reserved.

Mots clés : Ecologie, Elapidae, milieux anthropisés, Côte d'Ivoire.

\section{Studies of some ecological parameters of the Elapidae of anthropized environments of six cities of Ivory Coast}

\begin{abstract}
Elapidae are often incriminated in accidents with sometimes fatal outcome during untimely encounters with the man in Côte d'Ivoire. However, few studies on their ecology have been carried out in this country to define prophylactic measures against these accidents. To do this, some
\end{abstract}


anthropized environments of six cities of Côte d'Ivoire were subject of a regular herpetological monitoring from January 2014 to December 2015. During this period, hand captures and trapping allowed the inventory of five Elapidae species in the six cities. A total of 725 individuals were captured, of which 299 were divided into five species in fallows, 272 of four species in dwellings and 154 individuals of three species in plantations. This ophidian fauna is characterized by two constant species and three accidental species. In terms of preferential habitat, these five species are divided into three mixed species, one savanna species and one forest species. Four species are in the unrated category of the IUCN red list. Considering the nycthemeral moment of capture, Dendroaspis viridis was abundantly obtained during the day. These few ecological parameters on Elapidae, now better known, are of great interest for the evaluation of the epidemiological risk of Elapidae bites in the cities studied.

(C) 2019 International Formulae Group. All rights reserved.

Keywords: Ecology, Elapidae, anthropized environments, Côte d'Ivoire.

\section{INTRODUCTION}

L'expansion des villes et la réduction des milieux naturels ont amené l'homme au contact des animaux sauvages (Piédallu et al., 2016). Ces contacts créent souvent un problème de conservation de la biodiversité du fait des conflits homme-faune de plus en plus récurrents (Marchand, 2013). Ce problème se pose avec acuité entre l'homme et les ophidiens du fait de la crainte que les derniers inspirent (Nonga et Haruna, 2015). Les envenimations et létalités dues aux ophidiens ont été rapportées pour les villes d'Odienné, de Yamoussoukro et d'Aboisso, respectivement situées au Nord, au Centre et au Sud de la Côte d'Ivoire (Chippaux, 2002). $\mathrm{Ce}$ sont donc des agglomérations qui bien qu'urbanisées, abritent des espèces ophidiennes responsables de ces accidents (Djohan et al., 2010). Les Elapidae et les Viperidae sont les deux familles de serpents incriminés dans la plupart des accidents (Chippaux, 2006a, 2007); selon Sharma et al. (2016) et Chippaux (2007), les morsures de serpents en général et celle des Elapidae en particulier constituent une urgence médicale. L'étude de l'écologie des serpents de cette famille, combinée à des enquêtes épidémiologiques pourrait permettre de définir plus précisément le risque d'envenimation due aux Elapidae (Chippaux, 2005).

C'est dans cette optique que la présente étude se propose de fournir des informations sur quelques aspects de l'écologie des Elapidae en milieu anthropisé. Il s'agit dans ce travail de déterminer les moments et les lieux propices de contact des Elapidae avec l'homme.

\section{MATERIEL ET METHODES Milieu d'étude}

L'étude a été réalisée dans six Département (Aboisso, Bouna, Dabou, Djekanou, Odienné et Yamoussoukro). Le choix de ces localités est motivé par le fait que des études épidémiologiques antérieures ont révélé dans la plupart, des cas d'accidents causés par les Ophidiens. Ces localités appartiennent, selon la latitude, à trois zones climatiques et biomes différents (Figure 1). Chacun des six Département a été subdivisée en quatre sites représentés par des villes et des villages. Chaque site renfermait trois points de collecte de quatre hectares chacun, suivant un gradient d'anthropisation (habitations, plantations et jachères). Les sites d'Odienné et de Bouna sont dans la zone soudanaise. Ils sont respectivement drainés par les affluents du Sassandra et par la volta noire Le climat est le type soudanais avec deux saisons (une sèche et une pluvieuse). Quant à Djekanou et Yamoussoukro, ils sont implantés dans la zone guinéo-congolaise/soudanaise, avec un climat tropical subhumide à quatre saisons (deux sèches et deux pluvieuses). Ces sites sont traversés par le fleuve Bandama. Les sites d'Aboisso et de Dabou sont situés dans 
la zone guinéo-congolaise et ont un climat de type tropical humide à quatre saisons (deux sèches et deux pluvieuses). Elles sont arrosées par un système de lagunes et de fleuves côtiers dont la lagune Ebrié à Dabou, la Bia, le lac d'Ayamé et la lagune Eby à Aboisso (AkéAssi, 2002 ; Savané, 2010 ; Savané et Konaré, 2010).

\section{Matériel}

Un appareil GPS (Map source Garmin GPS 60cs) a servi pour la géolocalisation des points de captures.

Les captures se sont faites à l'aide de pièges à pots (goulot d'entrée de $5 \mathrm{~cm}$ de diamètre, longueur de $20 \mathrm{~cm}$, volume de 5 $\mathrm{dm}^{3}$ ) et une pince américaine (ouverture de la mâchoire de $10 \mathrm{~cm}$; longueur de 1,30 m) a servi pour la saisie de l'animal. Egalement, un crochet télescopique (Crochet en acier, long de 90 à $150 \mathrm{~cm}$ ) a été utilisé pour la fixation du cou du spécimen capturé. Des sacs (sac en toile avec coutures plates de sécurité sur le pourtour, double fermeture par corde et bande velcro, poignée de transport, de longueur 80 $\mathrm{cm}$ et de largeur $45 \mathrm{~cm}$ ) pour l'emballage des spécimens capturés. Des cages de transit ont servi à la mise en captivité momentanée des spécimens prélevés.. Des guides d'identification des serpents de l'Afrique de l'Ouest (Chippaux, 2006b ; Trape et Mané, 2006) ont été utilisés pour l'identification des espèces d'Elapidae échantillonnées. L'ordonnancement utilisé pour la liste systématique des espèces est conforme à celui utilisé par Zaher et al. (2009) et Chirio (2013).

\section{Méthodologie}

Dans chaque point de collecte (habitats), les captures se sont déroulées en moyenne pendant 24 jours de suite tous les mois, de janvier 2014 à décembre 2015. Dans le cadre de ce travail, des recherches en placeaux et en transect de Piquet et al., (2012) ont été utilisées. Un placeau d'une surface de $50 \mathrm{~m}^{2}$ a été placé et visité deux fois par jour (entre $9 \mathrm{~h}$ et $10 \mathrm{~h}$ et entre $19 \mathrm{~h} 30$ et 21 h). Il est déplacé chaque trois jours de façon à couvrir une plus grande surface des plantations et des jachères, soit un total de 182 placeaux par habitats.

Les recherches en transect se sont déroulées de 10 h à 12 h et 21 h à 22 h 00 , en utilisant un transect itinérant de $500 \mathrm{~m}$ dans chaque habitat. Les plages horaires de $9 \mathrm{~h}$ à 12 h et de 19 h 30 à 22 h ont été utilisées car la majorité des ophidiens rentrent en activité à ces périodes du nycthémère selon Trape et Mané (2006).

Des pièges à pots appâtés ont été posés dans les plantations et dans les jachères.

Le fond de chaque dispositif est appâté avec des rats de laboratoire ou des œufs de poule domestique. Dans chacune des plantations et des jachères investiguées, neuf pièges à pot ont été disposés (18 pièges par sites), soit un total de 432 pièges pour l'ensemble des 24 sites. Ces pièges espacés de cinq mètres les uns des autres sont placés sur les côtés et le centre d'un quadra de $100 \mathrm{~m}^{2}$ de surface.

Chaque semaine, et dans chaque habitat, le quadra est déplacé de façon aléatoire de sorte à couvrir toute la surface à échantillonner. Ils ont été visités quotidiennement, entre $7 \mathrm{~h}$ et $8 \mathrm{~h}$ et entre $18 \mathrm{~h}$ et $19 \mathrm{~h}$ pour éviter que les animaux ne meurt dans les pièges

A chaque visite, les individus capturés ont été mis en captivité pour éviter les reprises Tous les individus gardés en captivité ont été relâchés à la fin de l'étude dans le milieu d'origine ou semblable dans un souci de conservation.

\section{Expressions mathématiques et analyses statistiques des données}

Les résultats obtenus expriment la richesse spécifique des milieux investigués et donnent pour chacune des espèces leur abondance, leur fréquence relative respective et leur degré d'occurrence. Les indices de diversité de Shannon, d'équitabilité et de similarité ont été calculés. Pour chacune des espèces, l'habitat préférentiel et le statut de conservation ont été déterminés.

La diversité spécifique des sites a été comparée à l'aide du test de Kruskal-wallis, au seuil $\alpha=0,05$. La distribution des espèces selon les sites et le niveau d'anthropisation a été illustrée par des analyses factorielles de correspondances. Toutes ces analyses ont été réalisées avec le logiciel STATISTICA 7.1. 


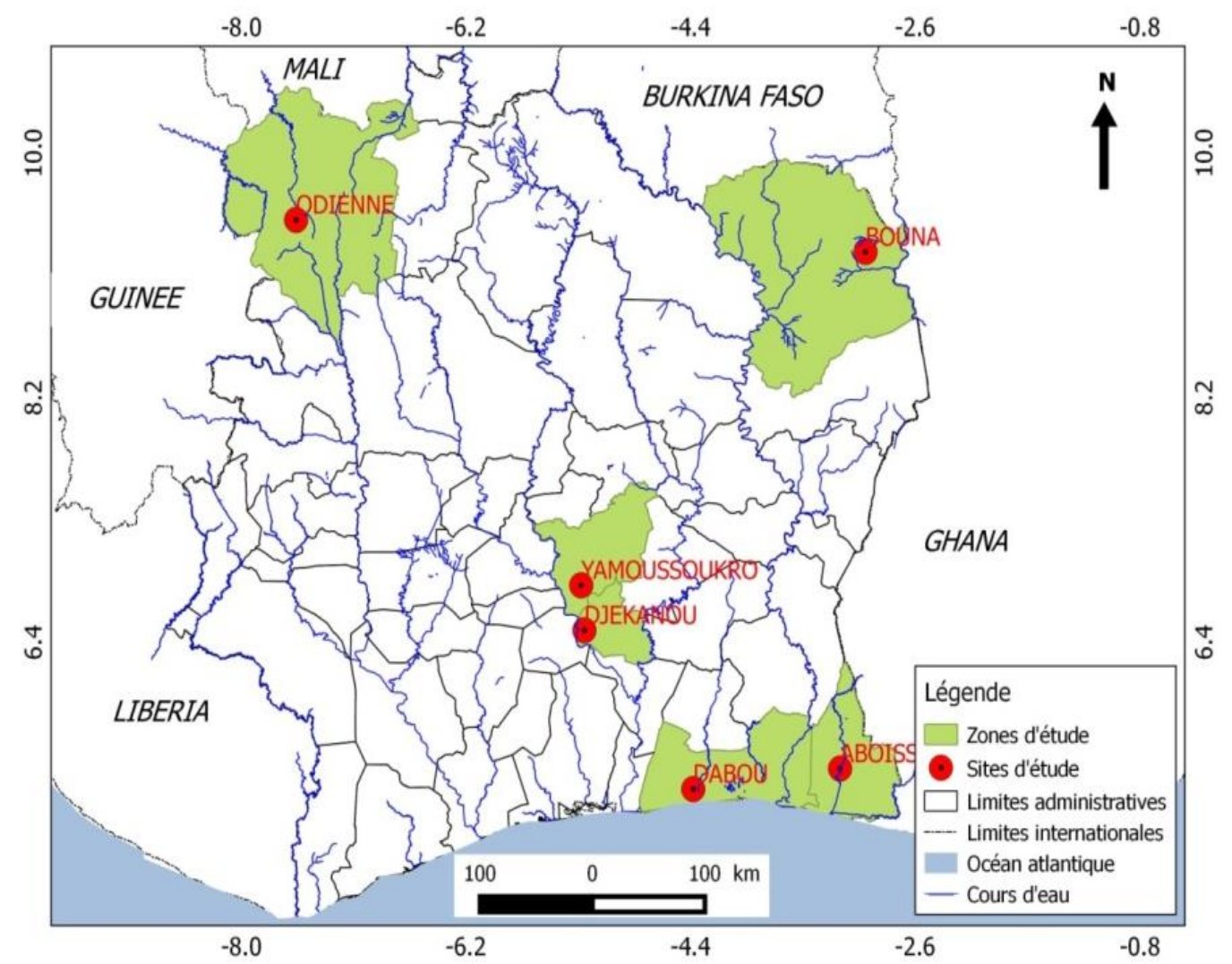

Figure 1 : Carte de Côte d'Ivoire indiquant les différents sites d'étude.

\section{RESULTATS}

Le suivi de la faune ophidienne des milieux anthropisés des six Départements a permis d'inventorier cinq espèces d'Elapidae dont quatre sont illustrées par la Figure 2. L'abondance relative globale est de 725 individus. Deux espèces, Dendroapis viridis (Hallowell, 1844) et Naja nigricollis Reinhardt, 1843 représentent ensemble $88,69 \%$ de l'effectif total, avec 643 individus.

Les trois autres espèces Elapsoidea semiannulata (Werner, 1897), Naja melanoleuca Hallowell, 1857 et Pseudohaje goldii (Boulenger, 1895), avec 82 individus, représentent ensemble $11,31 \%$ des effectifs (Tableau 1). L'indice de diversité (H') du peuplement global vaut 0,99 pour une équitabilité $(\mathrm{J})$ égale à 0,62 .

\section{Richesse spécifique et abondance relative des espèces d'Elapidae en fonction des sites}

Les sites d'Aboisso et de Bouna sont les plus riches avec quatre espèces d'Elapidae, soit une proportion de $80 \%$ de la richesse totale. Ils sont suivis par les sites de Dabou, de Djekanou, de Yamoussoukro et d'Odienné qui renferment chacun trois espèces d'Elapidae, soient $60 \%$ de la richesse spécifique des Elapidae observés (Tableau 1). Le test univarié de significativité basé sur le nombre d'espèces d'un site à l'autre indique qu'il y a effectivement une différence significative de la richesse spécifique des Elapidae d'un site à l'autre (test de Kruskalwallis ; $\mathrm{p}<0,05$ ).

Au niveau de l'abondance, le site de Bouna a renfermé le plus d'individus de serpents, avec 239 individus ( $\mathrm{Fr}=32,96 \%)$.

Il est suivi du site de Dabou, avec un effectif de 182 individus $(\mathrm{Fr}=25,10 \%)$. Le site de Yamoussoukro est le moins abondant en Elapidae, avec 38 individus dénombrés ( $\mathrm{Fr}$ $=5,24 \%$ ) (Tableau 1). Le test univarié de significativité basé sur l'abondance des espèces d'un site à l'autre indique qu'il y a effectivement une différence significative 
des abondances en Elapidae d'un site à l'autre (test de Kruskal-wallis ; $\mathrm{p}<0,05$ ).

L'analyse de la diversité écologique est illustrée par la Figure 3. Ainsi, il apparait que le site d'Aboisso est le plus diversifié, avec des indices de Shannon et d'équitabilité respectifs de 1,11 et 0,80 . Il est suivi des sites de Bouna, de Dabou, de Djekanou et de Yamoussoukro qui sont peu diversifiés, avec des valeurs d'indices de Shannon variant entre 0,90 et 1,04 . Enfin, le site d'Odienné est le moins diversifié $\left(\mathrm{H}^{\prime}=0,7 ; \mathrm{J}=0,64\right)$.

La distribution des Elapidae n'est pas homogène sur l'ensemble des six sites étudiés. Elle a été matérialisée par une Analyse Factorielle de Correspondance (AFC) qui prend en compte le nombre d'individus par espèce et par localité. A l'analyse du graphique (Figure 4) résultant de l'AFC, l'axe 1 qui a la plus forte inertie $(57,84 \%)$ permet de distinguer deux ensembles.

Le premier est représenté par les sites d'Aboisso, Dabou et Djekanou qui sont corrélés positivement à l'axe 1 , auxquels sont associés Naja melanoleuca (Najme) et Pseudohaje goldii (Psego). Le deuxième ensemble comprend les sites de Bouna et d'Odienné qui sont corrélés négativement à l'axe 1. A ces sites, sont associées les espèces Naja nigricollis (Odienné), Dendroaspis viridis et Elapsoidea semiannulata (Bouna).

\section{Richesse spécifique et abondance relative suivant le gradient d'anthropisation}

Les jachères des six sites prospectés ont abrité 299 individus $(\mathrm{Fr}=41,24 \%)$ pour l'ensemble des espèces d'Elapidae échantillonnées. Elles sont suivies des habitations qui renferment quatre espèces d'Elapidae, soit $80 \%$ de la richesse spécifique et 272 individus $(\mathrm{Fr}=37,52 \%)$. Les plantations, moins riches, comptent 154 individus $(\mathrm{Fr}=21,24 \%)$ appartenant à trois espèces d'Elapidae (Figure 5). Trois espèces d'Elapidae (Dendroapis viridis, Naja nigricollis et Naja melanoleuca) d'un effectif de 718 individus $(\mathrm{Fr}=99,03 \%)$ ont été collectées dans tous les milieux prospectés. Une espèce, Pseudohaje goldii, avec deux individus $(\mathrm{Fr}=0,27 \%)$ a été inventoriée dans une jachère et dans une habitation. En revanche, Elapsoidea semiannulata avec cinq individus $(\mathrm{Fr}=0,70 \%)$ a été collecté uniquement dans les jachères (Tableau 1).

En considérant le niveau d'anthropisation, l'analyse de la Figure 6 montre que l'axe 1 qui a la plus forte inertie $(92,62 \%)$ permet également de définir deux ensembles. Le premier est représenté par les fermes et les habitations qui lui sont corrélées positivement et auxquelles sont associées Naja melanoleuca (Najme), Naja nigricollis (Najni) et Pseudohaje goldii (Psego). Le deuxième ensemble, corrélé positivement, comprend les plantations et les jachères auxquelles sont associées les espèces Dendroaspis viridis et Elapsoidea semiannulata.

La diversité de ces milieux, illustrée par la Figure 7 montre que les jachères sont les plus diversifiées, avec des indices de Shannon et d'équitabilité respectifs de 1,05 et 0,65 . Elles sont suivies des plantations et des habitations qui sont peu diversifiées avec des valeurs d'indices de Shannon de 0,87 et 0,83 . Enfin, le site d'Odienné est le moins diversifié $\left(H^{\prime}=0,7 ; J=0,64\right)$.

\section{Similarité entre les sites}

L'analyse à classification ascendante hiérarchisée (Figure 8) réalisée au moyen de l'abondance relative des espèces d'Elapidae par site met en évidence trois groupes de sites bien hiérarchisés. Le premier groupe est composé de quatre sites (Aboisso, Yamoussoukro, Djékanou et Odienné). Cependant, dans ce groupe, il y a un regroupement des sites deux à deux. Le second groupe, constitué du site de Dabou, est distant du premier groupe par une distance d'agrégation de 27 . Le troisième groupe est formé par le site de Bouna qui est démarqué des deux premiers groupes par une distance d'agrégation comprise entre 60 et 70 .

\section{Caractérisation du peuplement d'Elapidae}

Suivant le degré de présence, deux catégories d'espèces ont été mises en évidence. La première est formée de deux espèces constantes (643 individus; $\mathrm{Fr}=$ $88,69 \%$ ) ayant pour degré de présence respective $67,46 \%$ et $50,73 \%$. La seconde 
catégorie est formée de trois espèces accidentelles (82 individus; $\mathrm{Fr}=11,31 \%$ ) dont les degrés de présence sont comprises entre $0,36 \%$ et $13,78 \%$. Les espèces constantes sont représentées par le Cobra cracheur Naja nigricollis et le Mamba vert Dendroaspis viridis. Les espèces accidentelles sont le Cobra noir et blanc Naja melanoleuca, 1' Elapsoide semiannélé Elapsoida semiannulata et Pseudohaje goldii (Tableau 1).

En ce qui concerne le statut de conservation, la quasi-totalité des espèces d'Elapidae inventoriées sont dans la catégorie non-évalué (NE) selon UICN (2017) excepté Dendroaspis viridis qui est dans la catégorie préoccupation mineur (LC). Au niveau des habitats préférentiels, le peuplement peut être subdivisé en trois groupes. Le groupe dominant est représenté par trois espèces ubiquistes qui contribuent pour $60 \%$ à la richesse spécifique totale.

Une espèce, Elapsoida semiannulata, est inféodée aux milieux savanicoles tandis

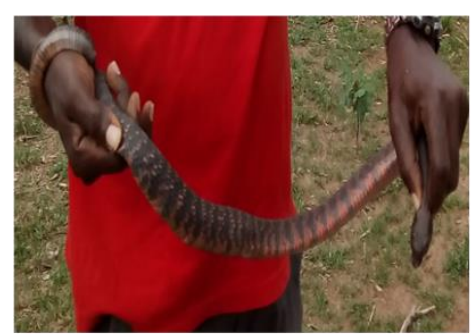

( a )

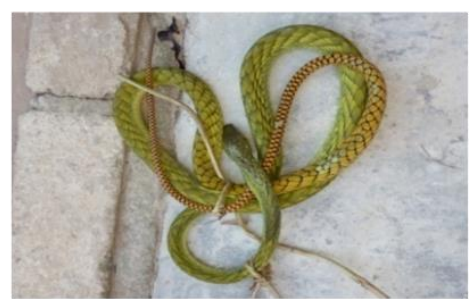

(c) qu'une autre, Pseudohaje goldii a été capturé en milieu forestier.

\section{Variation nycthémérale de la richesse spécifique et de l'abondance des Elapidae}

$\mathrm{Au}$ cours des 24 mois de suivi, la richesse spécifique des Elapidae était maximale le jour (cinq espèces) et minimale (trois espèces) à la tombée de la nuit. L'effectif diurne des Elapidae était quasiment le double (477 individus) de l'effectif nocturne (248 individus). Cependant, ces différences constatées ne sont pas statistiquement significatives (test de Kruskalwallis; $\mathrm{p}>0,05)$. Cette variation nycthémérale des effectifs est significative chez Dendroaspis viridis, avec 271 individus observés la journée, soit $56,81 \%$ des effectifs diurnes (test de Kruskal-wallis; $\mathrm{p}<0,05$ ). Chez Naja nigricollis le nycthémère n'influence pas significativement ses effectifs (test de Kruskal-wallis ; $p>0,05$ ) malgré les 220 individus obtenus la nuit, soit $88,71 \%$ des effectifs nocturnes (Figure 9).

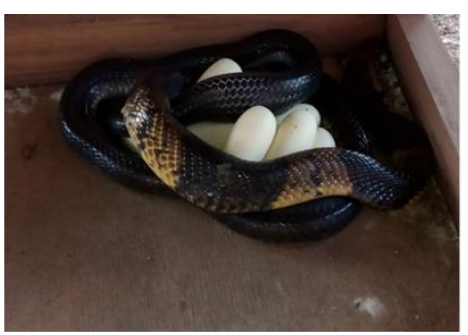

( b )

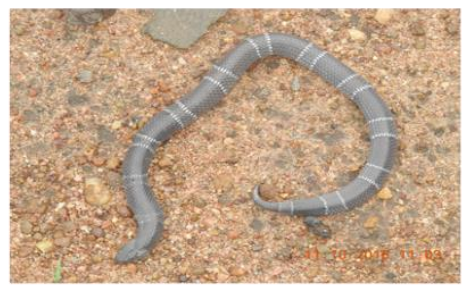

(d)

Figure 2 : Quelques représentants d'Elapidae a- Naja nigricollis; b- Naja melanoleuca $c$-Dendroaspis viridis et $d$-Elapsoidea semiannulata. 
Tableau 1: Liste phylogénétique des espèces d'Elapidae observées sur l'ensemble des sites et leur abondance respectives de janvier 2014 à décembre 2015.

\begin{tabular}{|c|c|c|c|c|c|c|c|c|c|c|c|c|c|c|}
\hline Espèces & $\begin{array}{l}\text { Nom } \\
\text { français }\end{array}$ & Abo & Bou & Dab & Dje & Odi & Yak & HP & Arg & $\operatorname{Fr}(\%)$ & $\operatorname{DP}(\%)$ & Car & St & Mi \\
\hline $\begin{array}{l}\text { Elapsoidae } \\
\text { semiannulata }\end{array}$ & $\begin{array}{l}\text { Elapsoide } \\
\text { semiannelé }\end{array}$ & & 5 & & & & & $S$ & 5 & 0,69 & 0,92 & Acci & $\mathrm{NE}$ & $\mathrm{J}$ \\
\hline $\begin{array}{l}\text { Naja } \\
\text { nigricollis }\end{array}$ & $\begin{array}{l}\text { Cobra } \\
\text { cracheur }\end{array}$ & 34 & 119 & 82 & 59 & 60 & 13 & M & 367 & 50,62 & 67,46 & Cons & $\mathrm{NE}$ & HJP \\
\hline $\begin{array}{l}\text { Naja } \\
\text { melanoleuca }\end{array}$ & $\begin{array}{l}\text { Cobra noir } \\
\text { et blanc }\end{array}$ & 12 & 11 & 35 & 12 & 1 & 4 & $\mathrm{M}$ & 75 & 10,34 & 13,78 & Acci & $\mathrm{NE}$ & HJP \\
\hline $\begin{array}{l}\text { Dendroaspis } \\
\text { viridis }\end{array}$ & $\begin{array}{l}\text { Mamba } \\
\text { vert }\end{array}$ & 31 & 104 & 65 & 23 & 32 & 21 & $\mathrm{M}$ & 276 & 38,07 & 50,73 & Cons & LC & HJP \\
\hline $\begin{array}{l}\text { Pseudohaje } \\
\text { goldii }\end{array}$ & $\begin{array}{l}\text { Cobra } \\
\text { arboricol }\end{array}$ & 2 & & & & & & F & 2 & 0,28 & 0,36 & Acci & $\mathrm{NE}$ & $\mathrm{HJ}$ \\
\hline
\end{tabular}

\section{Totaux}

$\begin{array}{llllll}79 & 239 & 182 & 94 & 93 & 38\end{array}$

725100

Abo : Aboisso ; Bou : Bouna ; Dab : Dabou ; Dje : Djekanou ; Odi : Odienné ; Yak : Yamoussoukro ; Arg : Abondance relative générale ; Fr : Fréquence relative ; HP : Habitat préférentiel ; Car : Caractérisation suivant le degré de présence ; St : Statut UICN ; Mi : Milieu de capture ; Acci : Accidentelle ; Cons : constante ; J : Jachères ; HJP : Habitations, Jachères et Plantations ; HJ : Habitations et Jachères ; NE : Non évalué ; LC : Préoccupation mineur ; DP : Degré d' occurrence.

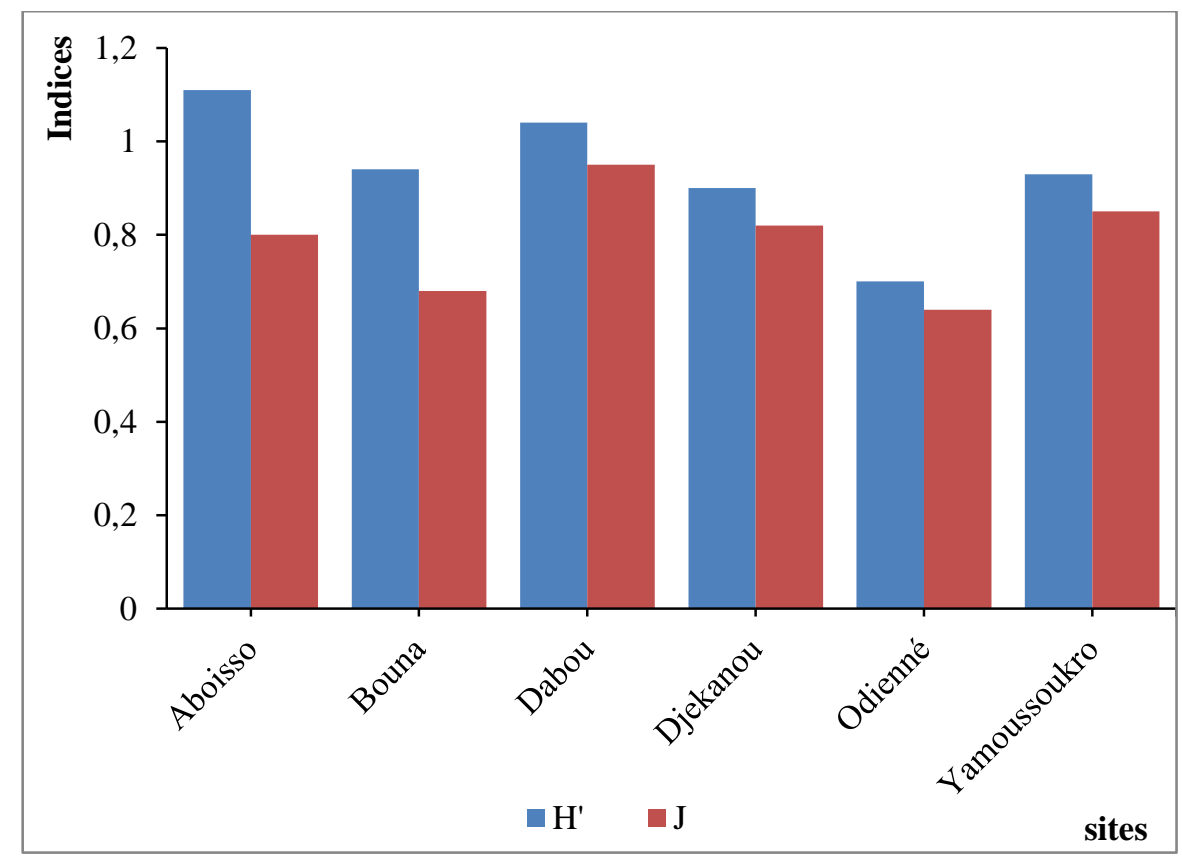

Figure 3 : Variations des indices de diversité de Shannon-Weaver (H') et d'équitabilité (J) des espèces d'Elapidae recensées selon les sites. 


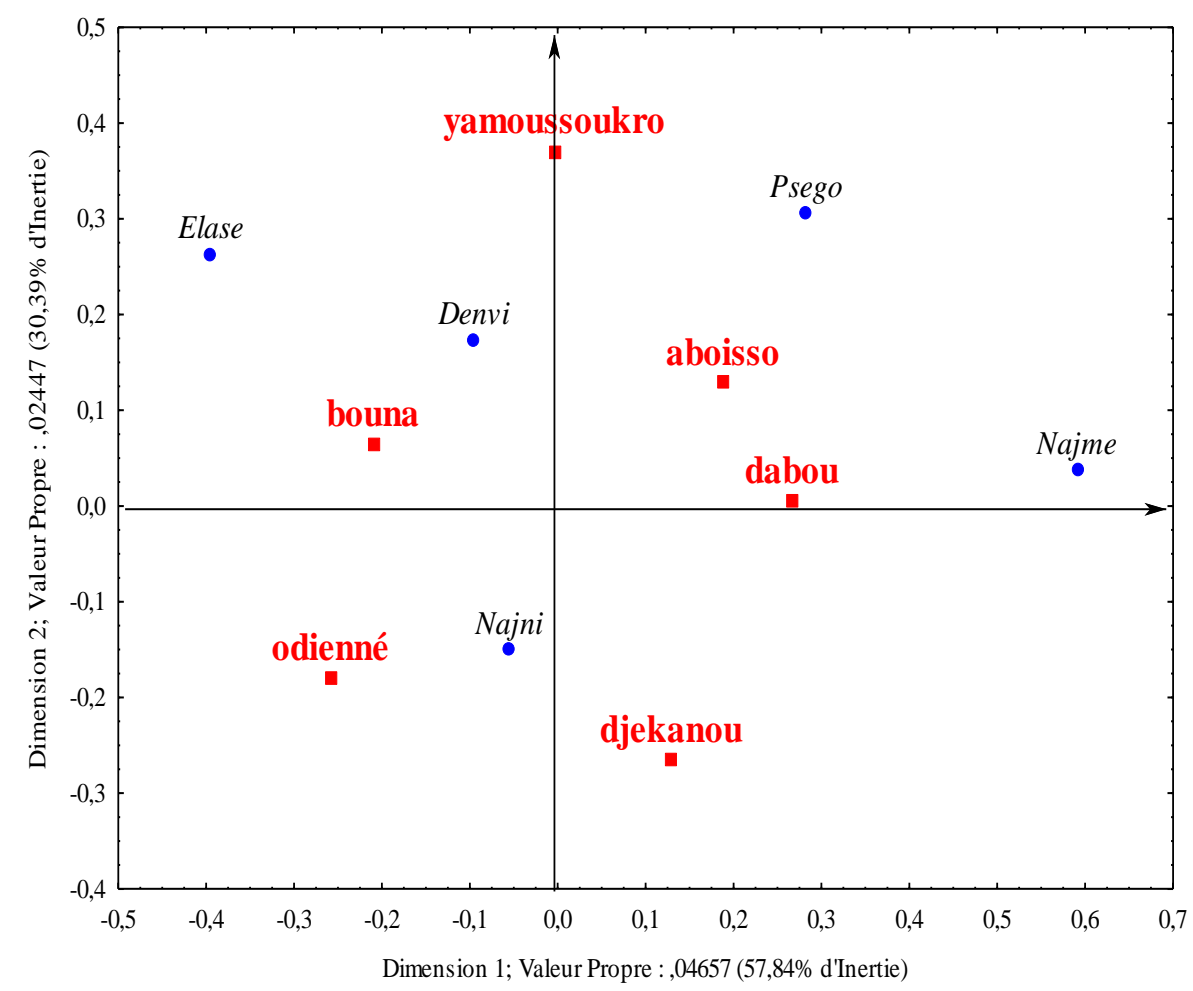

Figure 4: Distribution des espèces d'Elapidae en fonction des six sites étudiés.

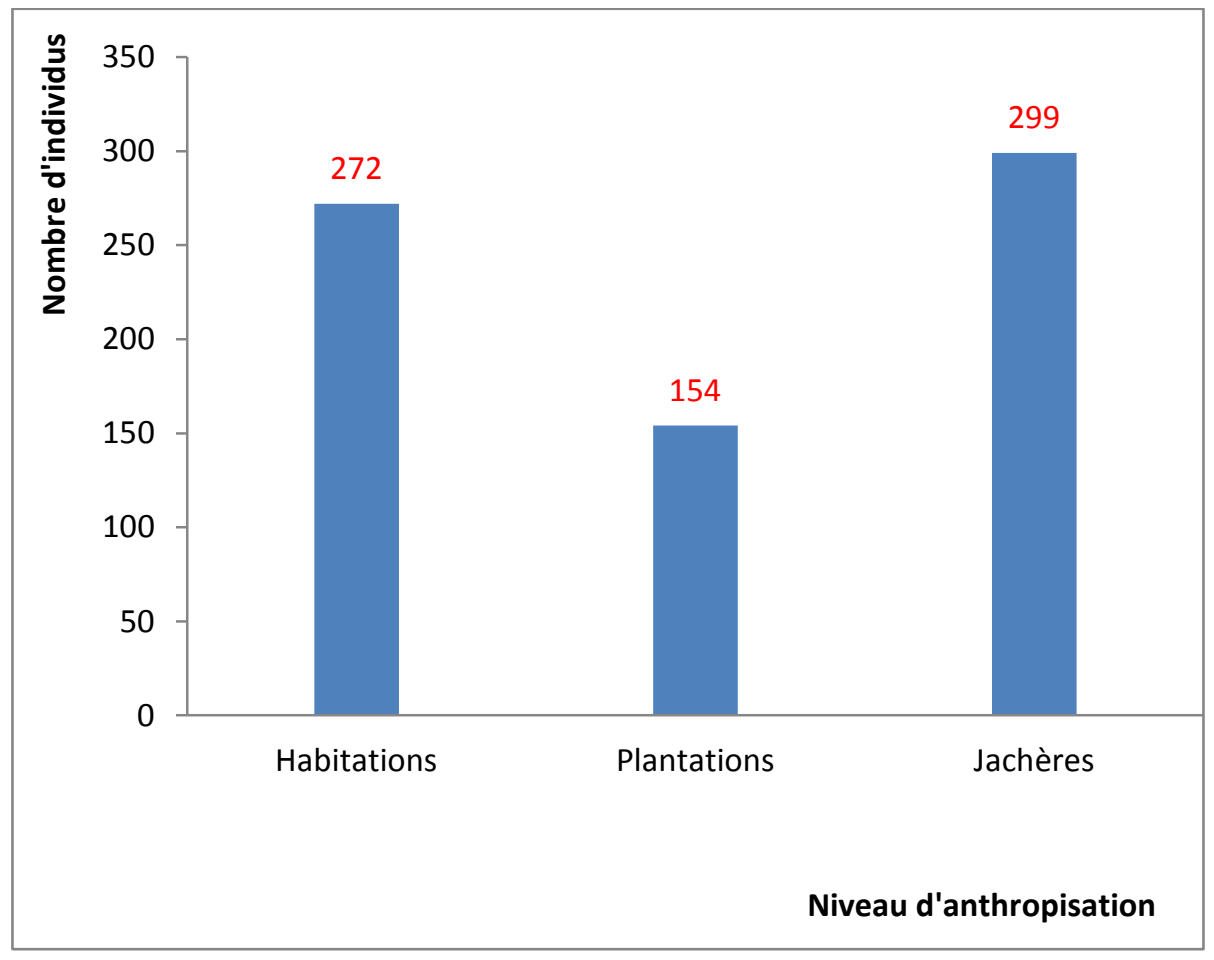

Figure 5 : Abondance des Elapidae selon le gradient d'anthropisation. 
M. H. AKAFFOU et al. /Int. J. Biol. Chem. Sci. 13(1): 237-250, 2019

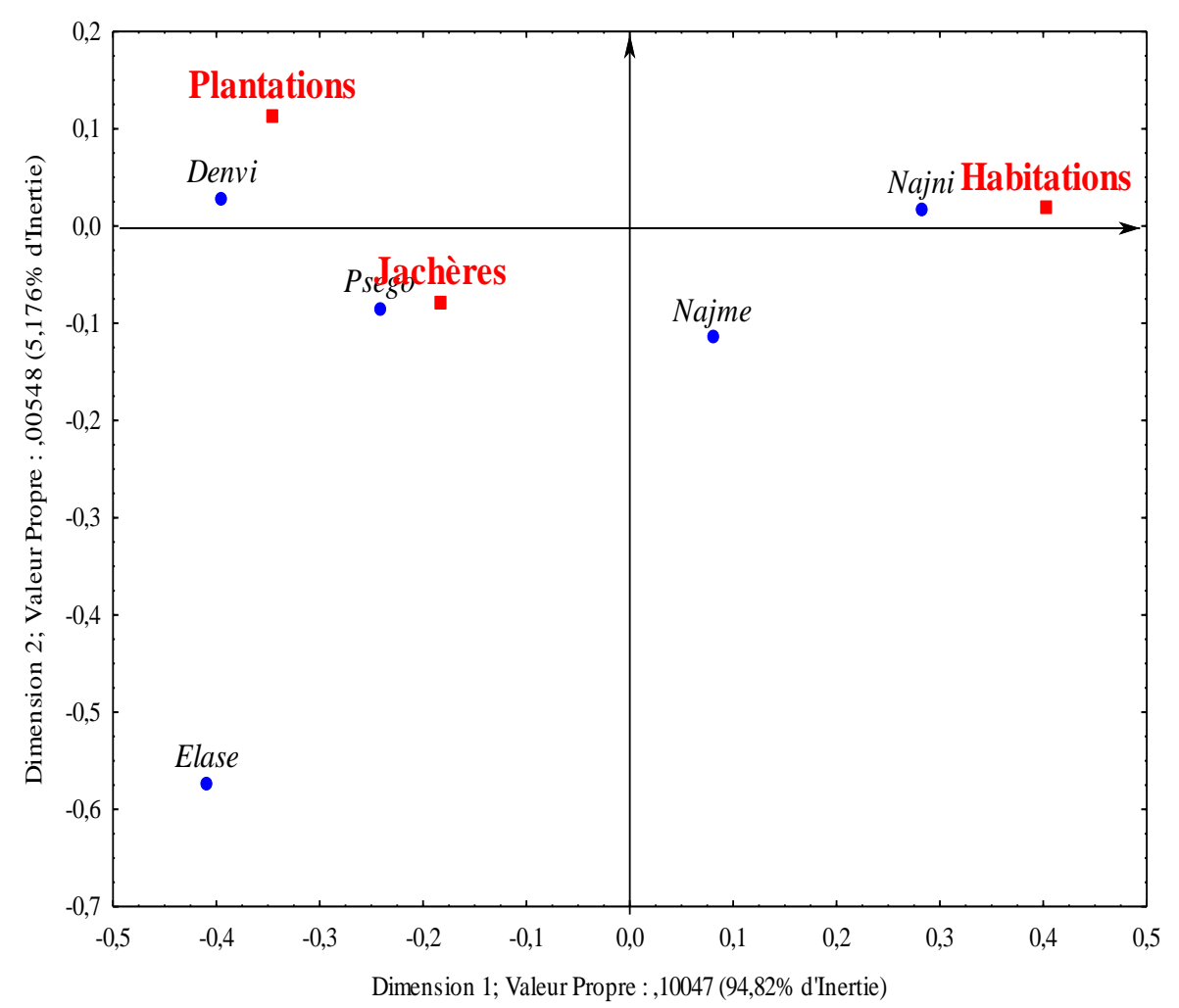

Figure 6: Distribution des espèces d'Elapidae selon le niveau d'anthropisation.

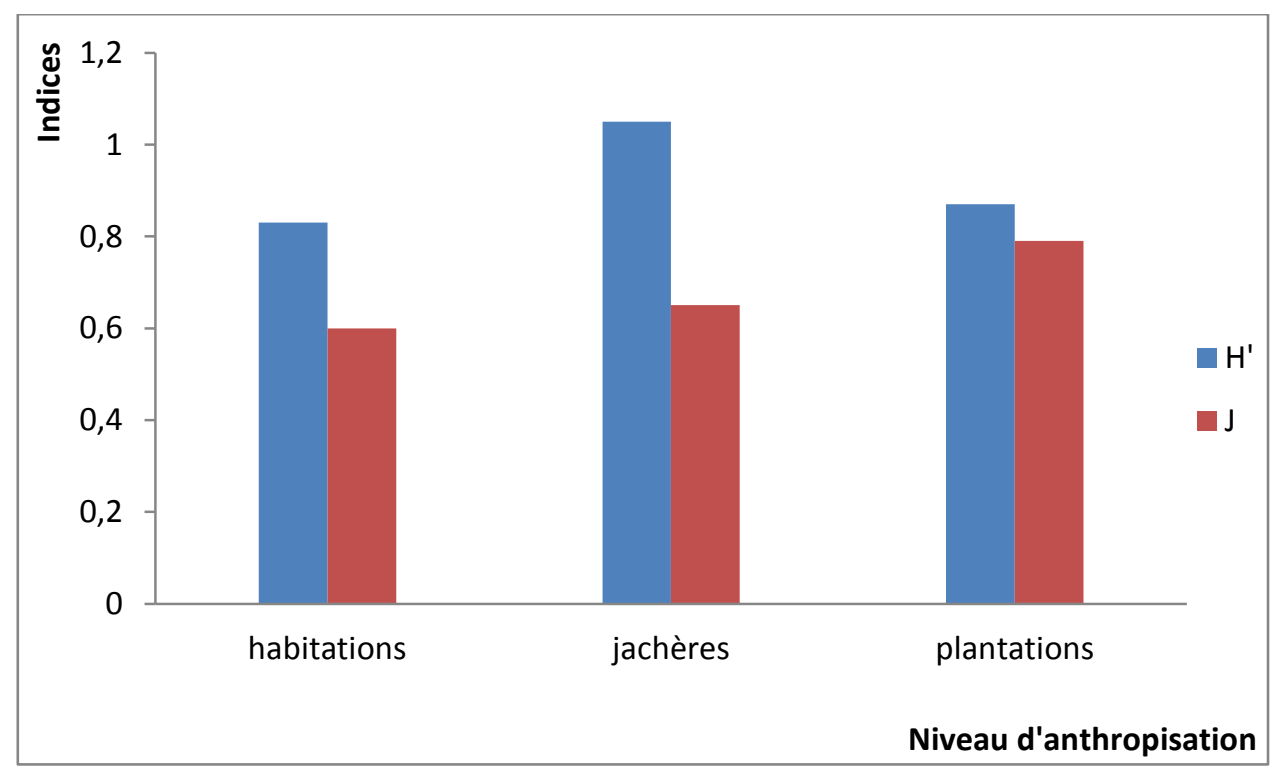

Figure 7 : Variations des indices de diversité de Shannon-Weaver (H') et d'équitabilité (J) des espèces d'Elapidae recensées selon le niveau d'anthropisation. 


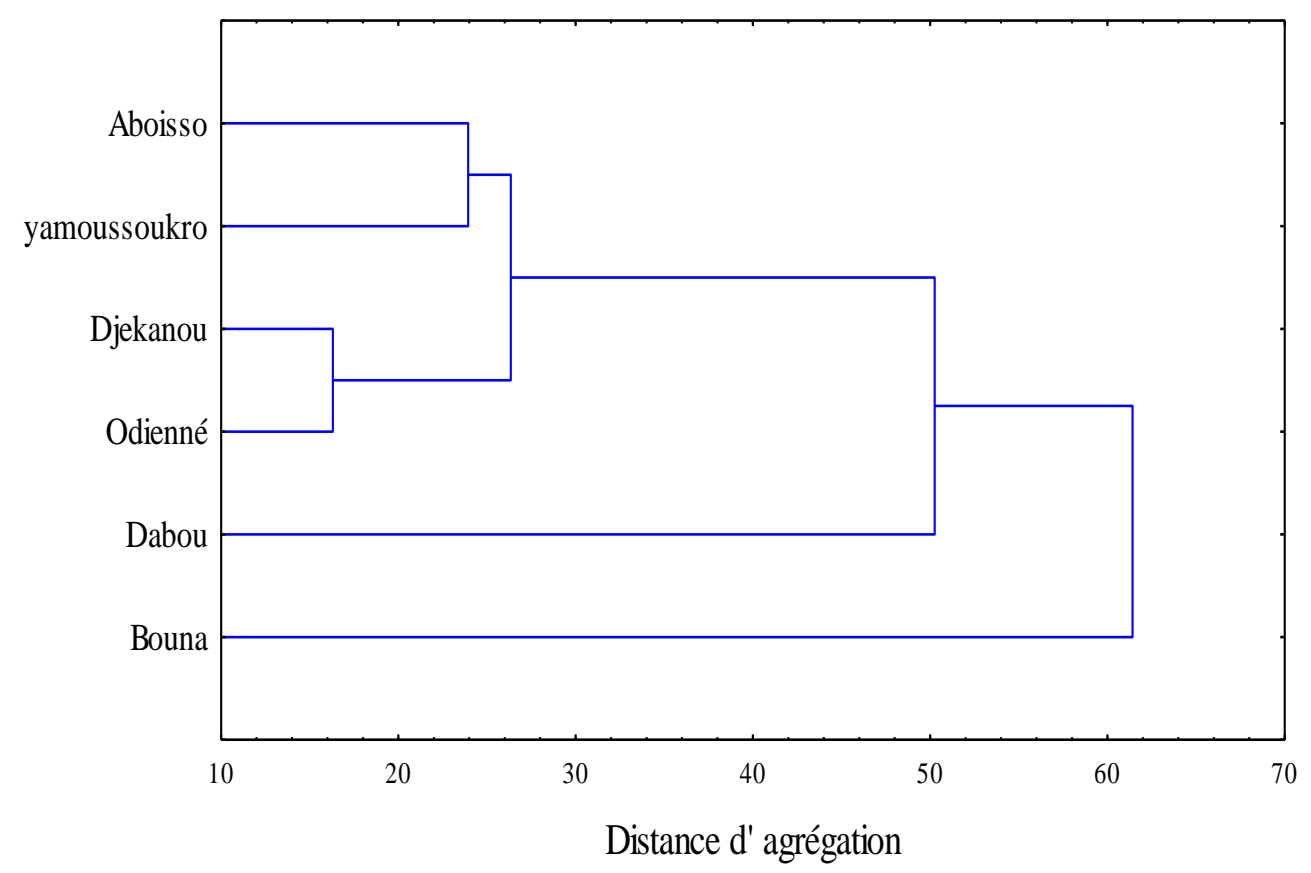

Figure 8 : Dendrogramme exprimant les niveaux de similarité entre le peuplement d'Elapidae de six sites de Côte d'Ivoire.

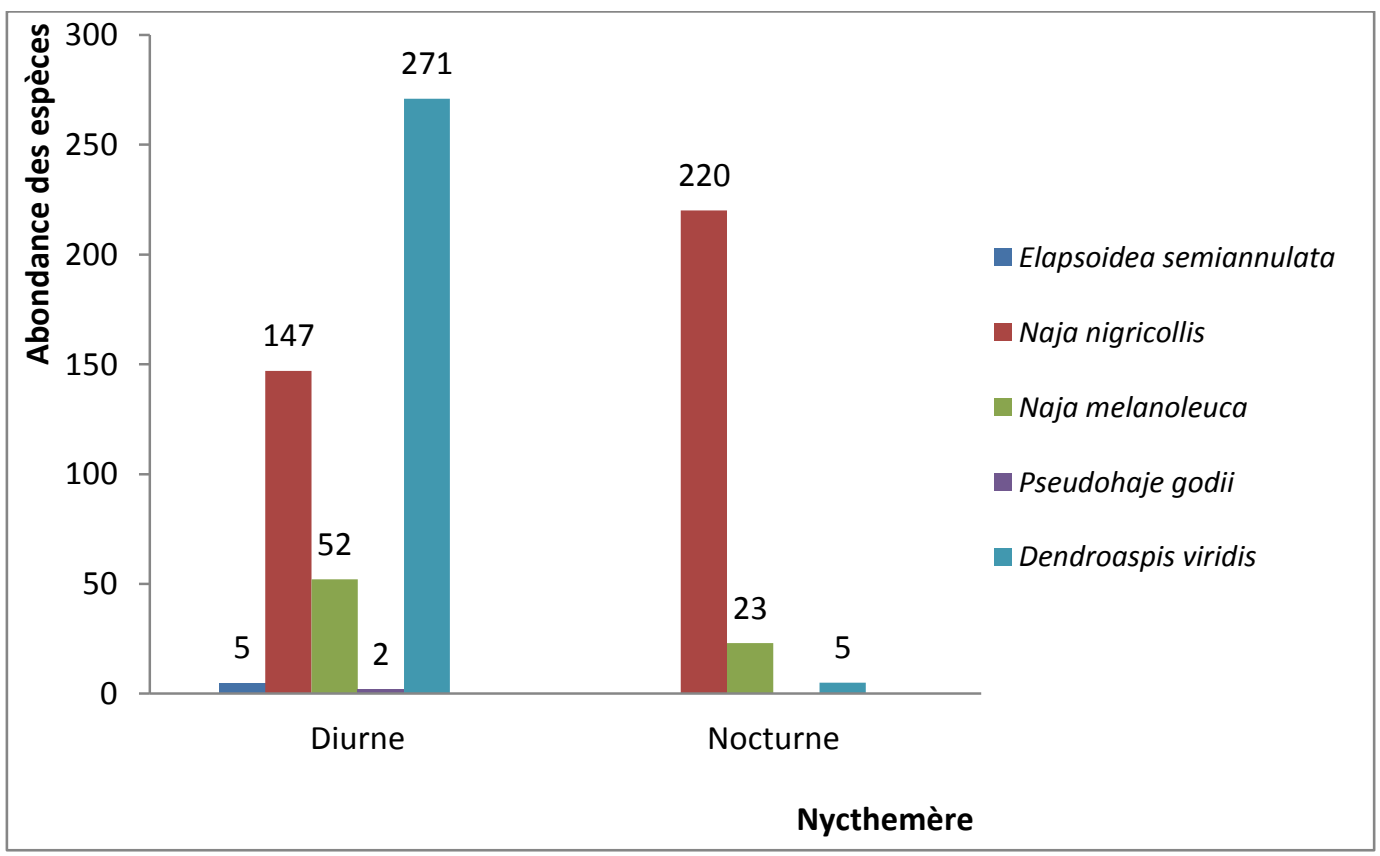

Figure 9 : Variations du peuplement d'Elapidae suivant l'activité nycthémérale. 


\section{DISCUSSION}

Cette étude dresse, pour la première fois, la liste des espèces d'Elapidae visitant quelques milieux anthropisés de six localités de Côte d'Ivoire. Cette diversité est supportée par plus de la moitié du nombre d'espèce (cinq espèces, fréquence relative $=62,5 \%$ ) de la composition spécifique totale (huit espèces d'Elapidae) connue de Côte d'Ivoire (Trape et Baldé, 2014). Ce nombre important d'espèces observées, témoignerait du fait que les milieux anthropisés investigués constituent des sites attractifs pour certaines espèces d'Elapidae.

L'absence dans cette étude de Naja katiensis est dûe au fait que les différents sites ne couvrent pas son aire de répartition; en plus, l'espèce est très peu commensale et fréquente très peu la zone soudanaise (Trape et Mané, 2006).

Certaines espèces, comme Dendroaspis polylepis et Pseudohaje goldii sont peu fréquentes (Chippaux, 2006b). Ce qui expliquerait le faible nombre d'individus de Pseudohaje goldii obtenu.

D'autres travaux effectués dans des milieux anthropisé, montrent que les espèces d'Elapidae obtenues dans la présente étude sont les plus communément observées. En effet, la combinaison des résultats des travaux d'Akaffou et al. $(2017,2018)$ montre que trois des espèces d'Elapidae collectées (Dendroaspis viridis, Naja melanoleuca et Naja nigricollis) sont les plus communes des milieux anthropisés de Côte d'Ivoire. Par contre, Rödel et Mashberg (2000) ont obtenus deux de ces espèces, Dendroaspis viridis et Naja melanoleuca, dans le Parc national de Taï (Côte d'Ivoire); Piquet et al. (2012) mentionnent également deux espèces d'Elapidae (Naja melanoleuca et Naja sp.) au Benin. Ces résultats se justifieraient d'une part , par le fait que ces travaux se sont déroulés uniquement en milieu forestier contrairement aux nôtres qui prennent en compte tous les faciès écologiques de Côte d'Ivoire et par le fait que ces milieux étant protégées, la végétation dense n'aurait pas facilitée la capture d'un plus grand nombre d'espèces ophidiennes d'autre part. Ailleurs, en Guinée, Chirio (2013) a montré que ces espèces
d'Elapidae, observées au cours de cette étude, sont les plus communes d'Afrique de l'Ouest. Ainsi, Akani et al. (2007) font mention de quatre espèces d'Elapidae (Dendroaspis jamesoni, Naja nigricollis, Naja melanoleuca et Pseudohaje goldii) dans les plantations de palmiers à huile au Nigéria.

Les espèces d'Elapidae ne sont pas toutes ubiquistes; cela expliquerait pourquoi elles n'ont pas été toutes capturées dans tous les habitats prospectées.

En effet, certaines d'entre elles ont des exigences écologiques qui les éloignent des écosystèmes fortement remaniés par l'homme ; c'est ainsi que Rödel et Mashberg (2000) montraient que les espèces de Pseudohaje nigra ne se rencontrent que dans les habitats forestiers.

Cependant, les cinq espèces inventoriées au cours de cette étude se seraient adaptées à la présence humaine du fait de leur capture dans des habitats conquis par l'homme. Elles pourraient donc être considérées comme des espèces anthropophiles dont il est important de suivre la démographie de leurs populations pour minimiser les contacts accidentels Hommeserpent.

Les cobras cracheurs (Naja nigricollis) et mambas verts (Dendroaspis viridis) sont les plus abondants du peuplement d'Elapidae obtenus sur l'ensemble des sites. Cette remarquable abondance des espèces s'expliquerait par leur grande valence écologique et leur importante capacité à se reproduire (Chippaux, 2006b). Par ailleurs, Lougbégnon et al. (2009) justifie que ces abondances sont dues au fait que ces espèces se seraient très bien adaptées à divers biotopes. L'importance numérique de Naja nigricollis dans les milieux anthropisés a également été rapportée par Akaffou (2004) à Abidjan où Naja nigricollis était l'espèce numériquement la plus importante du peuplement des Ophidiens récoltés.

La distribution spatiale du peuplement des Elapidae varie d'un site à l'autre ; ainsi, les sites de forêt et de zone pré forestières (Aboisso, Dabou et Djekanou) regroupent deux espèces (Naja melanoleuca et 
Pseudohaje goldii) qui ont en commun leur appartenance à cette zone écologique (Luiselli et al., 2002 ; Chirio, 2013).

A l'opposé, les sites de savane qui selon Diffo (2001) offrent les meilleures conditions de vie aux reptiles (nourriture disponible, tolérance physiologique, compétitions intra et interspécifiques moindres, prédation moins poussée, etc.), ont abrité la plupart des espèces d'Elapidae de savane comme Dendroaspis viridis, Naja nigricollis et Elapsoidea semiannulata.

S'agissant de la distribution des espèces suivant le niveau d'anthropisation, les espèces du genre Naja sont proches des habitations tandis que les espèces des genres (Dendroaspis et Elapsoïdea) préfèrent les plantations et les jachères.

Les caractéristiques des habitations (végétations non entretenues) ainsi que certaines activités humaines (élevage de poules domestiques, entassement d'ordures ménagères) offriraient des proies potentielles et des gîtes favorables à leur maintien dans ce milieu (Akaffou et al., 2018). Chippaux (2006b) observait également que Naja nigricollis est très proche de l'homme, voire commensale et abonde à proximité des habitations pour se nourrir de rongeur et d'oiseaux, parfois d'œuf de poule. D'autres auteurs, observaient que l'espèce pénètre dans les cours des maisons à la recherche de volaille et d'œufs (Blanc et Fretey, 2000 ; Trape et Mané, 2006). Ces auteurs observaient aussi que les espèces des genres Dendroaspis et Elapsoïdea ont une préférence pour les plantations et les jachères qui leur offrent leur habitat naturel (les espèces des genres Dendroaspis étant arboricoles tandis que celles du genre Elapsoidea sont fouisseuses).

Les variations nycthémérales d'abondance étaient beaucoup plus importantes chez Dendroaspis viridis (tendance diurne) et chez Naja nigricollis (tendance nocturne). Ces observations pourraient être corrélées à leur écologie alimentaire. En effet, chez la première espèce (Dendroaspis viridis), la tendance diurne pourrait s'expliquer par le fait que lors de nos capture, cette espèce se nourrissait de lézards, de rats palmistes (que nous observions aussi le jour) et d'oisillons dans leur nid (en l'absence des parents en quête de nourriture le jour).

En revanche, les individus de Naja nigricollis se nourrissaient de poussins, de Lissamphibiens ou encore des rats; ces proies (Lissamphibiens et rongeurs) ont été observées pendant nos travaux au crépuscule ; cela expliquerait la tendance nocturne de cette espèce. Les tendances diurne ou nocturne des espèces susmentionnées ont été rapportées par différents auteurs. Ainsi, Dendroaspis viridis est présenté comme une espèce héliophile (Trape et Mané, 2006). Quant à Naja nigricollis, ce serait une espèce aux mœurs nocturnes (Chippaux, 2006b ; Akaffou et al., 2017).

\section{Conclusion}

Ce travail qui visait l'inventaire des Elapidae des milieux anthropisés de six Département de Côte d'Ivoire ainsi que les lieux et les moments potentiellement favorables aux rencontres Homme - Elapidae fait état de la présence de cinq espèces dans ces milieux. Ces rencontres inopportunes seraient plus probables avec Dendroaspis viridis, le jour, dans les plantations et les jachères et avec les espèces du genre Naja dans les habitations, la nuit. L'amélioration de nos connaissances sur ces facteurs écologiques permet de contribuer à l'évaluation du risque épidémiologique par les Elapidae pour ces six localités étudiées. Ces données aideraient également les responsables sanitaires de ce pays à mieux cerner la lutte contre les morsures de serpents en Côte d'Ivoire.

\section{CONFLITS D'INTERETS}

Les auteurs déclarent qu'ils n'ont aucun intérêt en concurrence.

\section{CONTRIBUTIONS DES AUTEURS}

MHA est l'investigateur principal; MTKB a contribué à la rédaction de ce manuscrit; HKY-B a validé l'analyse des données et fournis des orientations dans la rédaction de ce manuscrit. Garant scientifique, 
conception du thème de recherche et supervision scientifique de l'étude.

\section{REMERCIEMENTS}

Nous tenons à remercier les Directeurs départementaux de la Santé, les chargés de l'information, les médecins-chefs, les infirmiers, les Agents de Santé Communautaire (ASC) et les autorités coutumières qui ont permis la réalisation de ces travaux. Nos remerciements vont à l'endroit du technicien animalier de l'Institut Pasteur de Côte d'Ivoire, M. Sawadogo Arouna qui a facilité la formation sur les techniques de capture aux ASC et à l'endroit de Messieurs Ouattara Hoborogni et Gue Gbiya Gauthier pour leur sollicitude. Nous remercions Adon Arsène pour son aide constante dans la rédaction de cet article.

\section{REFERENCES}

Akaffou MH, Chippaux JP, Allali KB, Coulibaly Z, Dosso M. 2017. Peuplement ophidien des plantations de Hevea brasiliensis d'Anguédédou (SudEst Côte d'Ivoire). Bull. Soc. Herp. Fr., 162: 31-38.

Akaffou MH, Allali KB, Coulibaly Z, Konan KL, Camara A, Yao KA, Sawadogo A, Dosso M. 2018. Inventaire préliminaire des serpents de la ville de Tengréla et ses alentours (Nord-Ouest, Côte d'Ivoire). REB-PASRES, 3(1): 36 - 43. DOI : https://rebpasres.org/wp-

content/uploads/2018/12/REB-PASRES31_AKAFFOU-et-al.pdf

Akaffou MH. 2004. Envenimations Ophidiennes à Abidjan: Principales espèces Ophidiennes récoltées et bilan du traitement de 51 cas de morsures de serpents en réanimation aux $\mathrm{CHU}$ de Cocody et Treichville. Thèse de Doctorat d'Etat en Pharmacie, Université Felix Houphouët Boigny d'Abidjan (Cocody), Abidjan, p.116.

Akani GC, Ebere N, Luiselli L, Eniang EA. 2007. Community structure and ecology of snakes in fields of oil palm trees (Elaeis guineensis) in the Niger Delta, southern Nigeria. Afr. J. Ecol., 46: 500-
506.

DOI:

https://doi.org/10.1111/j.1365-

2028.2007.00885.x.

Aké-Assi L. 2002. Flore de la Côte d'Ivoire: catalogue systématique, biogéographie et écologie. II. Boissiera, 58 : 401 pp.

Blanc PC, Fretey T. 2000. Les Reptiles de la réserve de faune de la Lopé et de la forêt des Abeilles (Gabon). Bull. Soc. Zool. Fr., 125(4): 281-292.

Chippaux JP. 2002. Épidémiologie des morsures de serpent en République de Côte d'Ivoire. Bull Soc. Pathol. Exot., 95(3): 167-171.

Chippaux JP. 2005. Évaluation de la situation épidémiologique et des capacités de prise en charge des envenimations ophidiennes en Afrique subsaharienne francophone. Bull Soc Pathol Exot., 98(4): 263-8.

Chippaux JP. 2006a. Envenimations et empoisonnements par les animaux venimeux ou vénéneux. II. Envenimations par Viperidae. Med. Trop., 66 : 423-428.

Chippaux JP. 2006b. Les Serpents de l'Afrique Occidentale et Centrale ( $3^{\text {ème }}$ édn). IRD : Paris.

Chippaux JP. 2007. Envenimations et empoisonnements par les animaux venimeux ou vénéneux : Envenimations par Elapidae. Med. Trop., 67: 9-12.

Chirio L. 2013. Inventaire des reptiles de la région de Sangarédi (Guinée maritime). Bull. Soc. Herp. Fr., 144: 67-100.

Diffo J. 2001. Etude de la distribution du peuplement reptilien du parc national de Bouba ndjidah (Nord-Cameroun), Mémoire DEA, Université de Yaoundé I, Cameroun, p.73.

Djohan V, Menan EI, Yavo W, Barro PC, Vanga H, Dempah JA, N'Dri DK, Mignonsin D, Akaffou MH, Koné K. 2010. Herpetological data and management of ophidian envenomation at university hospitals in Abidjan, Côte d'Ivoire. Med. Trop., 70(1): 99.

Lougbegnon OT, Tente, BA, Codja JTC, Libois MR. 2009. Distribution des passereaux dans les biotopes forestiers et 
de substitution forestiers au SudBénin.'Bulletin de la Recherche Agronomique du Bénin, 65: 52-71. DOI: http://dx.doi.org/10.4314/ijbcs.v4i4.6305 6.

Luiselli L, Akani GC, Corti C, Angelici MF. 2002. Is sexual size dimorphism in relative head size correlated with intersexual dietary divergence in West African forest cobras, Naja melanoleuca? Contrib. Zool., 71(4): 141145.

Marchand G. Les conflits hommes/animaux sauvages sous le regard de la géographie. Carnets de Géographes, mis en ligne le 01 janvier 2013, consulté le 22 janvier 2019. DOI: $10.4000 /$ cdg. 1070

Nonga HE, Haruna A. 2015. Assessment of human-snake interaction and its outcomes in Monduli District, northern Tanzania. Tanzania Journal of Health Research, 17(1): 1-12. DOI: http://dx.doi.org/10.4314/thrb.v17i1.9.

Piédallu B, Quenette PY, Mounet C, Lescureux N, Borelli-Massines $\mathrm{M}$, Dubarry E, Camarra JJ, Gimenez O. 2016. Spatial variation in public attitudes towards brown bears in the French Pyrenees. Biological Conservation, 197: DOI: 90-97. https://doi.org/10.1016/j.biocon.2016.02. 027.

Piquet A, Toudonou C, Konetché L, Sinsin B, Chippaux JP. 2012. Étude préliminaire de la faune ophidienne de la forêt classée de la Lama, Sud Bénin. Bull. Soc. Pathol. Exot., 105: 166-170. DOI : https://doi.org/10.1007/s13149012-0206-9.

Rödel MO, Mashberg D. 2000. Vorläufige liste der Schlangen des Taï Nationalparks Elfenbeinküste und angrenzender Gebiete. Salamandra, 36(1): 25-38.

Savané I. 2010. Principaux facteurs environnementaux de la Côte d'Ivoire: le climat. In: Konaté S \& Kampmann D, (eds) : Atlas de la Biodiversité de l'Afrique de l'Ouest. Biota, 3: 123-124.

Savané I, Konaré A. 2010. Principaux facteurs environnementaux de la Côte d'Ivoire: le climat. In Konaté S \& Kampmann D, (eds) : Atlas de la Biodiversité de l'Afrique de l'Ouest, Biota, 3:123-124.

Sharma SK, Kuch U, Höde P, Bruhse L, Pandey DP, Ghimire A, Alirol E. 2016. Use of Molecular Diagnostic Tools for the Identification of Species Responsible for Snakebite in Nepal: A Pilot Study. PLoS Neglected Tropical Diseases, 10(4). DOI : e0004620. http://doi.org/10.1371/journal.pntd.0004 620.

Trape JF, Mané Y. 2006. Guide des Serpents d'Afrique Occidentale, Savane et désert. IRD : Paris.

Trape J-F, Balde C. 2014. A checklist of the snake fauna of Guinea, with taxonomic changes in the genera Philothamnus and Dipsadoboa (Colubridae) and a comparison with the snake fauna of some other West African countries. Zootaxa, 3900(3): 301-338. DOI: doi.org/10.11646/zootaxa.3900.3.1.

Zaher H, Grazziotin GF, Cadle EJ, Murphy WR, Cesar de Moura-Leite J, Bonatto LS. 2009. Molecular phylogeny of advanced snakes (Serpentes, Caenophidia) with an emphasis on South American Xenodontines: a revised classification and descriptions of new taxa. Papéis Avulsos de Zoologia, 49(11): $\quad 115-153 . \quad$ DOI: doi.org/10.1590/S003110492009001100001. 